\title{
EFFECTS OF A PATENT DUCTUS ARTERIOSUS ON POSTPRANDIAL INTESTINAL BLOOD FLOW IN EXTREMELY LOW BIRTH WEIGHT (ELBW) NEONATES
}

\author{
T. Havranek ${ }^{1}$, M. Rahimi ${ }^{2}$, E. Armbrecht ${ }^{3}$ \\ ${ }^{I}$ Pediatrics/Neonatology, ${ }^{2}$ Pediatrics/Cardiology, ${ }^{3}$ Center for Outcome Research, Saint Louis University, \\ St.Louis, MO, USA
}

Background: Patent ductus arteriosus (PDA) affects superior mesenteric artery blood flow velocity (SMA $\mathrm{BFV}$ ) in fasting neonates. Recently, it has been reported that PDA affects postprandial mesenteric perfusion in premature baboons; however, the effects of PDA on postprandial mesenteric perfusion in preterm neonates have not been evaluated.

Aims: : To determine whether PDA affects postprandial SMA BFV in ELBW neonates.

Methods: Appropriate for gestational age, ELBW preterm neonates, tolerating bolus enteral feedings were enrolled in this prospective observational study. Pulsed Doppler was used to measure preprandial and postprandial (at 30-min and 60-min) time-averaged mean, peak and end diastolic velocities (TAMV, PSV, EDV) once during the day of life 5 to 7 ; at the same time, PDA size was estimated using the PDA:left pulmonary artery (LPA) ratio : 0.5 (i.e., moderate/large PDA) and $<0.5$ (i.e., small/no PDA). Mean values across time were graphed to illustrate relative changes in moderate/large and no/small PDA groups. Repeated measures ANOVA was used to compare SMA BFV changes between the groups from preprandial to 30-min and 60-min postprandial for each type of measured velocity (TAMV, PSV, EDV).

Results: Of the 38 infants studied, 16 were in the no/small PDA group; 22 were in the moderate/large PDA group. Postprandial TAMV, PSV and EDV were not different between these two groups ( $\mathrm{p}$ values were $0.798,0.315$ and 0.59 , respectively).

Conclusions: Changes in postprandial mesenteric blood velocity did not differ between infants with no/small PDA versus moderate/large PDA in this study's cohort of 38 ELBW neonates. 